\title{
Targeting the Spinal Nerve via A Double-Needle, Transforaminal Approach in Failed Back Surgery Syndrome: DEMONSTRATION OF A TECHNIQUE
}

\author{
Rinoo V. Shah, MD, Wesley Merritt, MD, Dwayne Collins, RN, and Gabor B. Racz, MD
}

Spinal surgery, particularly spinal fusion surgery, alters the anatomy of the spine and hence, may increase the difficulty of performing an interventional spine procedure. Transforaminal epidural procedures have gained popularity as an alternative to interlaminar epidural steroids in the management of radicular pain syndromes. Patients

Failed back surgery syndrome (FBSS) is a syndrome characterized by the persistence of pain following spinal surgery for a benign, spinal pain condition (1). Interventional pain techniques may be useful in this population in terms of diagnosis and therapy, but there is one caveat: surgically induced alterations in spinal anatomy may increase the difficulty in performing procedures correctly and safely. Interlaminar epidural steroid injections, epiduroscopy, and epidural adhesiolysis with decompressive neuroplasty have a demonstrable safety and feasibility record in FBSS (1-5).

The feasibility and safety record of transforaminal epidural and spinal nerve injections have been alluded to in two studies $(6,7)$. Geurts et al $(6)$ evaluat-

From Department of Anesthesiology, Pain Division Texas Tech University Health Sciences Center, Lubbock, TX. Address Correspondence: Rinoo V. Shah, MD, Assistant Professor, Dept. of Anesthesiology, Pain Division, TTUHSC/ International Pain Institute, 4430 South Loop 289, Lubbock, TX 79414.

E-mail: rinoo_shah@yahoo.com

Support: The RK ${ }^{T M}$, RX-Coude ${ }^{T M}$, and curvedblunt nerve block needles used in this study were provided by EPIMED International, Inc., $141 \mathrm{Sal}$ Landrio Dr., Crossroads Business Park, Johnstown, NY 12095; the RFK ${ }^{T M}$ radiofrequency needle used in this study were provided by Radionics, 22 Terry Avenue, Burlington MA 01803; and Omnipaque $240 \mathrm{TM} \mathrm{mg} / \mathrm{dl}$ was provided by Nycomed Princeton NJ, 08540

Conflict of Interest: Dr. Gabor B. Racz is a consultant to Epimed Int. and Radionics Inc. However, no external funding was received for this study and no financial benefit will be conferred on the authors or on the device manufacturers mentioned herein. with failed back surgery syndrome are often excluded or represent a minor subset in many clinical studies evaluating the efficacy of transforaminal procedures. When included, however, patients with FBSS have typically undergone laminectomies or microdiscectomies: these procedures are less likely to violate the foraminal space in the spine

ed the efficacy of dorsal root ganglion radiofrequency lesioning in a large prospective, double-blind study, in which $50 \%$ of the patients had undergone prior spinal operations. Devulder (7) evaluated the efficacy of transforaminal epidural steroids with hyaluronidase in patients that had undergone a laminectomy. In both studies, a single needle technique was used, but a detailed description of the technique was left out. In any case, spinal nerve/transforaminal injections are typically reserved for patients with virgin spines (8-10).

Although comfortable with the use of these techniques on patients without prior spinal surgery, a practitioner may be reluctant to use them in FBSS, due to concerns about technical difficulties and complications. We demonstrate a technique for accessing the epidural space/ spinal nerve via a transforaminal approach that may be easily adopted.

\section{Demonstration of a Technique}

\section{Back Ground Information}

A 48 year-old, gainfully employed professional, was referred to our pain clinic for the management of a focal, leftsided, lower back pain. He had an unremarkable medical history apart from an allergy to penicillin. A history of chronic low back pain led to the performance of an L4-5, L5-S1 posterolateral and posterior lumbar interbody fusion with cages, approximately 10 years prior to pre- compared to fusion surgery. We describe the specific details of a transforaminal approach to the epidural space/spinal nerve in a patient with a posterolateral and posterior interbody fusion.

Keywords: Failed back surgery syndrome, transforaminal epidural, spinal nerve injection. sentation. He was pain free until one year prior to presentation. He began to develop pain just above the left posterior iliac crest, approximately at the level of Tuffier's line. His pain was described as "gnawing" and "aching." His pain was minimal with rest, but significant with mechanical loading activities, particularly standing. These episodes ranged from 7 to $8 / 10$ on the numerical pain rating scale (NRS-11). Non-steroidal anti-inflammatory analgesics provided no relief. He declined stronger analgesics due to concerns about cognitive impairment and sedation, both of which would interfere with his profession and active lifestyle. Imaging studies included radiographs that demonstrated interbody cages and a posterolateral fusion mass, at the aforementioned levels. There was no evidence of postjunctional degeneration, segmental instability, pseudoarthrosis, or cage dislodgement. Magnetic resonance imaging studies with and without contrast demonstrated post-operative changes consistent with mild post-operative scarring and artifact from the hardware. The lumbar nerve roots appeared adequately decompressed. There was only mild loss of signal and disc height at L3-4. These films were reviewed extensively by the chief of spinal surgery at a premier academic medical center and a referral to a pain specialist was made.

The first pain specialist performed blockade of the medial branch nerves of the left posterior L1, L2, L3 primary rami. The patient had no relief. A second pain 
specialist performed left-sided L3, L4, and L5 spinal nerve injections, using a transforaminal approach, on separate days. The dictations, but not the fluoroscopic images of these procedures were available: these dictations simply stated that a double needle technique was used and the needle was advanced into the corresponding neural foramen. The patient kept detailed anatomic drawings of the analgesic response after each procedure. The $\mathrm{L} 3$ and L5 spinal nerve injections provided no relief. The L4 spinal nerve injection provided $>80 \%$ reduction in the pain for several hours. The patient sought further consultation with his spine surgeon and was sent for a bone scan. There was increased uptake in an area around the fusion mass on the left side around the L4 and L5 levels. He was subsequently referred to our pain clinic for evaluation.

On physical exam he was lean and in good shape. The neurological exam was normal. Adverse neural tension signs were absent. There was no position or provocative maneuver that could reproduce his pain. Inspection demonstrated a midline and left-sided iliac crest scar. His stated that his pain was located a few centimeters above the iliac crest scar, approximately 5 $6 \mathrm{~cm}$ left of midline.

We offered to inject this area with local anesthetic and steroid for diagnostic purposes. The area was actually marked with an indelible marker and the injection was performed under fluoroscopic guidance. The needle was advanced until bone contact was made at the level of the fusion mass. The patient reported concordant reproduction of his low back pain during the injection. After the procedure, the patient reported a $>80 \%$ relief of pain for a few hours.

We then obtained a CT scan with reconstruction and fine cuts through the left sided fusion mass. There appeared to be a pseudoarthrosis in the fusion mass that was shaped like an "L." In other words, one limb of the pseudoarthrosis extended from the dorsal to the mid-portion of the fusion mass in a sagittal plane. The second limb took a leftward, 90-degree turn from the first and was oriented in the coronal plane. We then scheduled a local anesthetic and steroid injection into the pseudoarthrosis under CT guidance. The dorsal limb of the pseudoarthrosis was easily accessible and proper needle placement was confirmed with contrast enhancement. Concordant pain was repro- duced during the injection and near total pain relief was obtained for 24 hours.

We then referred him for consultation with a neurosurgeon investigating the role of bioabsorbable materials in the management of pseudoarthroses in lumbar spine surgery. Another neurosurgeon advised placing screws into the fusion mass to augment stability. Finally, the patient sought consultation with a third neurosurgeon, who concurred on revising the pseudoarthrosis. During surgery, this neurosurgeon felt the fusion mass was stable, but found the L3-4 level to be unstable. Hence, the fusion was extended to L3-4, with pedicle screws, intradiscal cages, and plates. This surgery had no impact on the patient's pain symptoms following recovery.

Approximately one year after our first interventional diagnostic procedure, the patient returned to our clinic seeking further options for treatment. He declined neuromodulatory or implantable procedures. Epidural adhesiolysis and decompressive neuroplasty were offered, but this procedure was denied by the insurance company. The rationale for this procedure was based on the patient's his- tory of a significant analgesic response to the L4 spinal nerve injection.

We offered a repeat block as a prelude to performing pulsed radiofrequency lesioning of the L4 dorsal root ganglion/ spinal nerve. The patient, however, desired pulsed radiofrequency lesioning to be done, irrespective of a repeat block. The patient agreed with this plan of treatment.

\section{Description of the Procedure}

Informed consent was obtained. The patient was placed in a prone position with a pillow underneath the abdomen to reduce the lumbar lordosis. Standard American Society of Anesthesiology monitors were used and oxygen was administered by nasal cannula. Standard sterile preparation was performed. The patient declined any intravenous sedation. An anteroposterior fluoroscopic image was obtained. The pedicle/pedicle screw of the left L4 vertebral body was located to identify the left L4-5 intervertebral foramen. A horizontal line was drawn laterally through the lower portion of the foramen. We then drew a vertical line that demarcated the outer edge of the left-sided

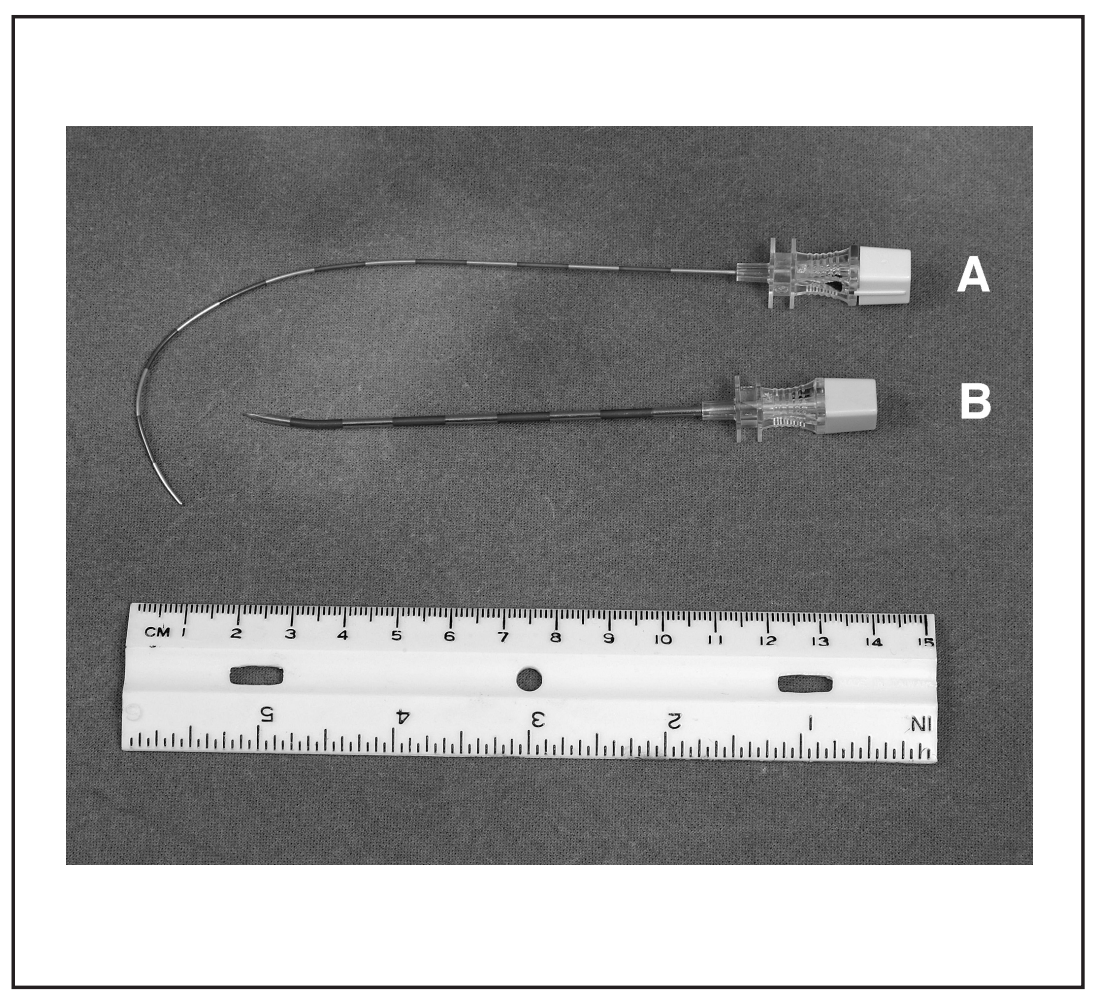

Fig 1. (A) A $15 \mathrm{~cm}, 20$ gauge, curved-blunt nerve block needle (Epimed Int.) with a distal ' $J$ ' curve; (B) A 3.5", 15-gauge RX-CoudeTM (Epimed Int.) needle. The needle is fed through the introducer. 


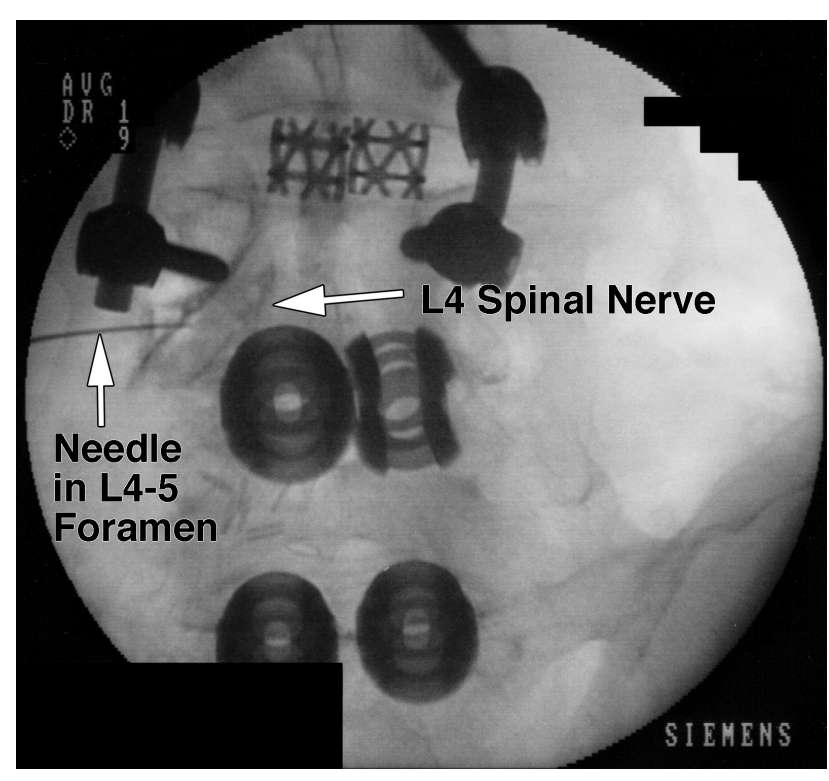

Fig 2. The $15 \mathrm{~cm}$ length, $15 \mathrm{~mm}$ active tip, curved-blunt RFK ${ }^{T M}$ (Epimed Int.) needle is advanced into the left L4-5 intervertebral foramen in an anteroposterior fluoroscopic projection. A dual contrast outline of the nerve and transforaminal and cephalic contrast spread is demonstrated. The fusion mass, cages, and hardware are visualized.

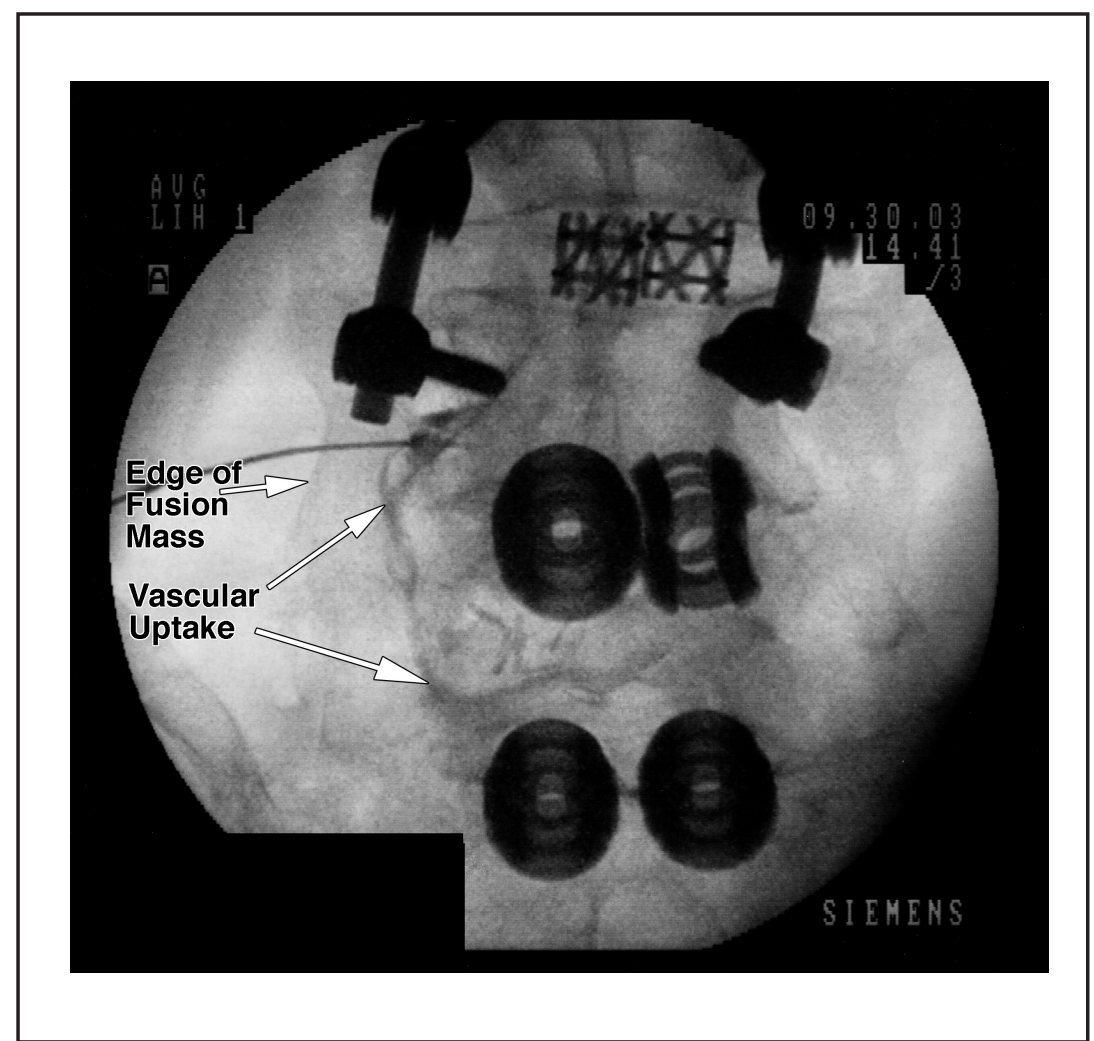

Fig 3. A better delineation of the fusion mass is on the antero-posterior view is seen. Real-time contrast instillation demonstrates vascular uptake. lateral mass fusion. This vertical line was approximately 5 centimeters left of midline. The C-arm was then obliqued approximately $30-40$ degrees, such that this vertical line now overlay the lateral edge of the L4 vertebral body. Due to the presence of an interbody fusion, the degree of the cephalic-caudad angle was a 'best' guess to align the endplates. We raised a skin wheal with $1 \%$ lidocaine and a 27 gauge needle, approximately two centimeters to the left of where these two lines intersected. The C-arm was obliqued until the vertical line was flush with the edge of the vertebral body. A $31 \frac{1}{2}$ " 16-gauge introducer was advanced in a gun-barrel fashion through the skin wheal, just past the edge of the fusion mass. A lateral view confirmed that the tip of the introducer was past the posterior elements, but dorsal to the intervertebral foramen. An antero-posterior view, however demonstrated that the introducer was fairly lateral to the foramen. A blunt-curved 20gauge, $15 \mathrm{~cm} \mathrm{RFK}^{\mathrm{TM}}$ radiofrequency needle with a $15 \mathrm{~mm}$ active tip was curved distally, to approximate the shape of a "J." This needle was fed through the introducer. The needle was advanced just to the tip of the introducer. At that point, the introducer was slowly withdrawn, while simultaneously advancing the needle. This allowed increased medial movement and minimized anterior movement of the radiofrequency needle. Furthermore, the degree of anterior movement was minimized, since the J-tip curled back posterior-wards. For demonstration purposes, a rigid 3"-3.5" 15 or 16-gauge introducer needle can be been substituted for the angiocatheter and $a \geq 4.5$ " 20 -gauge curved blunt nerve block needle can be substituted for the radiofrequency probe (Fig. 1) when doing nerve blocks. The amount of curvature needed, for the nerve block or radiofrequency needle, will depend on how lateral the introducer is to the foramen. The RFK needle was advanced medially into the mid-upper portion of the L4-5 foramen. Non-ionic, water soluble, iodinated contrast (Omnipaque ${ }^{\mathrm{TM}} 240$ $\mathrm{mg} / \mathrm{dl}$ ) was instilled. A dual contrast outline of the L4 spinal nerve, along with cephalic, transforaminal spread of contrast medial to the left L4 pedicle, confirmed proper placement (Fig. 2). Real-time fluoroscopy, however, demonstrated vascular uptake (Fig. 3). The needle was re-positioned twice, with the same vascular pattern. This pattern was confirmed on a lat- 


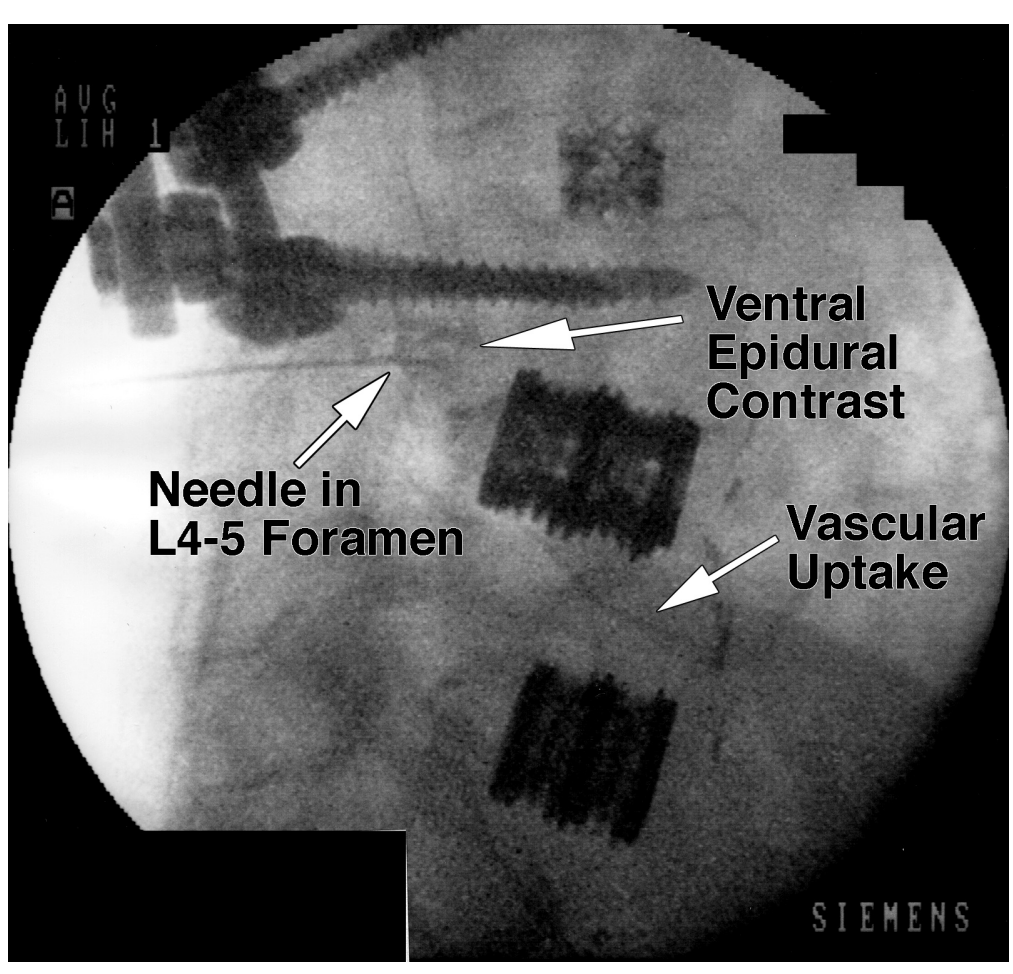

Fig 4. A lateral fluoroscopic projection demonstrates optimal needle placement in the L4-5 foramen. Vascular uptake is again demonstrable.

eral fluoroscopic projection, along with ventral epidural spread (Fig. 4). We decided against placing local anesthetic or steroid. We initiated sensory testing at 50 Hertz and paresthesias in the L4 dermatomal distribution occurred at 0.45 Volts. The paresthesias overlapped with his area of pain. Motor testing was initiated at 2 Hertz and L4 myotomal contractions occurred at 1 Volt. Due to this 2 -fold dissociation in sensory-motor recruitment, we proceeded with 4 cycles of pulsed radiofrequency lesioning: each cycle consisted of a pulse width of 20 milliseconds, a frequency of $2 \mathrm{Hertz}$, and a constant temperature of 42 degrees Celsius. The respective impedance and voltage outputs at the initiation of the first, second, third, and fourth cycles were, respectively, as follows: 250 ohms and 69 Volts, 210 ohms and 54 Volts, 198 ohms and 50 Volts, and finally, $194 \mathrm{ohms}$ and 47 Volts. These impedance measurements suggested that we were perineural and not intraneural. Additionally, the decrement in impedance and voltage has been consistent with our clinical experience in pulsed radiofrequency lesioning of spinal nerves/dorsal root ganglia. The needle was removed. The neu- rological exam post-procedure was normal. The patient reported concordant reproduction of pain that persisted for one week. He did not obtain any pain relief at 6-weeks. A repeat appeal was made to the medical director of his insurance company and an epidural adhesiolysis and decompressive neuroplasty procedure has been approved. The patient is pending this procedure.

\section{Discussion}

Schofferman et al (11) characterizes FBSS as "a non-specific term that implies that the final outcome of surgery did not meet the expectations of both the patient and the surgeon that were established before surgery." Long et al (12) proposed several etiological subgroups based on poor patient selection, poor clinical judgment, poor surgical technique, and the development of post-surgical complications. Some practitioners may consider this to be an oversimplification. From a surgical perspective there are many additional reasons as to why patients 'fail' spinal surgery and in turn, these reasons mitigate the culpability of the operating surgeon: inadequate decompression, progressive spinal stenosis, persistent radiculopathy with perineural scarring, spinal stenosis adjacent to lumbar fusion, failed discectomy, pseudoarthrosis, iatrogenic instability, subjacent disc degeneration with discogenic pain, foraminal stenosis, post-decompression deformity and spinal stenosis by natural history $(11,13,14)$.

Magnetic resonance imaging is the optimal exam for most cases of FBSS, but CT-scanning with multi-planar reformatting is preferred for evaluating a pseudoarthrosis (11). Nonetheless, even with these imaging modalities, the identification of a specific etiology in FBSS remains elusive (15). Many of these etiologies suggest that revision surgery is necessary to confirm or refute the diagnosis. One caveat is that surgery and imaging do not provide the patient with an opportunity to provide feedback in terms of pain relief. Herein lay the promising role of an interventional pain procedure for the diagnosis and treatment FBSS. Anesthetic diagnostic injections may be valuable to establish if nerve root compression or inflammation is causing pain (11). Unfortunately, clinical trials that evaluate the efficacy of interventional spine procedures $(8,10)$, with the exception of those evaluating dorsal root ganglion radiofrequency lesioning (6), epidural adhesiolysis $(2,3)$, spinal cord stimulation (16), and continuous neuraxial medication delivery (17), typically exclude or have small numbers of FBSS patients. Interlaminar and caudal approaches to the epidural space have been safely demonstrated in patients with prior back surgery $(2-4,9)$.

We have successfully applied this technique to a number of patients with failed back surgery syndrome, specifically those with posterolateral fusions and instrumentation. A single needle approach may suffice in those patients with FBSS following a laminectomy or microdiscectomy (7). The use of a blunt needle has increased our confidence in the performance of these procedures, since a few passages may be required for optimal needle placement. Some may contend that use of a double needle approach may increase the risk of delivering microscopic metallic shavings into neural or epidural tissues. This has been refuted with a Tuohy needle during combined spinal-epidural procedures (18). The introducers we use minimize these concerns: angiocatheters or $\mathrm{RK}^{\mathrm{TM}}$ or RX-Coude ${ }^{\mathrm{TM}}$ needles. The proximal edge, i.e., heel, of the RK or RX- 
Coude introducer orifice is rounded in the vertical plane but flat in a horizontal plane; the heel of the Tuohy needle orifice is shaped like a 'V', which can act as a pincer when transmitting catheters or needles (19).

\section{CONCLUSION}

We have successfully demonstrated a transforaminal technique in a patient with FBSS, specifically a patient who underwent multiple lumbar spine fusion surgeries. This technique has been used on a number of patients at our institution with technical success. Our hope is that this case report will induce other interventional pain physicians to discuss and investigate issues about the feasibility, safety, reproducibility, rationale, and efficacy of this particular technique. If this technique gains wider acceptance, then we will have yet another tool to aid patients with failed back surgery syndrome.

\section{Author Affiliation: \\ Rinoo V. Shah, MD \\ Assistant Professor \\ Dept. of Anesthesiology, Pain Division \\ Texas Tech University Health Sciences \\ Center / International Pain Institute \\ 4430 South Loop 289 \\ Lubbock, Texas 79414 \\ E-mail: rinoo_shah@yahoo.com}

\section{Wesley Merritt, MD}

Dept. of Anesthesiology, Pain Division Texas Tech University Health Sciences Center / International Pain Institute 4430 South Loop 289

Lubbock, Texas 79414

\section{Dwayne Collins, RN}

Dept. of Anesthesiology, Pain Division Texas Tech University Health Sciences Center / International Pain Institute 4430 South Loop 289

Lubbock, Texas 79414

Gabor B. Racz, MD

Grover E. Murray Professor and Chairman Emeritus International Pain Institute, Texas Tech University Health Sciences Center $36014^{\text {th }}$ Street Lubbock, TX 79430 E-mail: paula.brashear@ttuhsc.edu

\section{REFERENCES}

1. Shah RV, Ericksen JJ, Lacerte M. Interventions in chronic pain management. 2. New frontiers: Invasive nonsurgical interventions. Arch Phys Med Rehabil 2003; 84: S39-44.

2. Heavner JE, Racz GB, Raj P. Percutaneous epidural neuroplasty: Prospective evaluation of $0.9 \% \mathrm{NaCl}$ versus $10 \% \mathrm{NaCl}$ with or without hyaluronidase. Reg Anesth Pain Med 1999; 24:202-207.

3. Manchikanti L, Pampati V, Fellows B et al. Role of one day epidural adhesiolysis in management of chronic low back pain: A randomized clinical trial. Pain Physician 2001, 4:153-166

4. Shah RV, Heavner JE. Recognition of the subarachnoid and subdural compartments during epiduroscopy: Two cases. Pain Practice 2003; 3:321-325.

5. Fredman B, Nun MB, Zohar E et al. Epidural steroids for treating "failed back surgery syndrome:" Is fluoroscopy really necessary? Anesth Analg 1999; 88:367-372.

6. Geurts JW, van Wijk RM, Wynne HJ et al. Radiofrequency lesioning of dorsal root ganglia for chronic lumbosacral radicular pain: A randomised, double-blind, controlled trial. Lancet 2003; 361:21-26.

7. Devulder J. Transforaminal nerve root sleeve injection with corticosteroids, hyaluronidase, and local anesthetic in the failed back surgery syndrome. J Spinal Disord 1998; 11:151-154.

8. Lutz GE, Vad VB, Wisneski RJ. Fluoroscopic transforaminal lumbar epidural steroids: An outcome study. Arch Phys Med Rehabil 1998; 79:1362-1366.

9. Boswell MV, Hansen HC, Trescot AM et al. Epidural steroids in the management of chronic spinal pain and radiculopathy. Pain Physician 2003; 6:319-334.

10. Rosenberg S, Grabinsky A, Kooser C et al. Effectiveness of transforaminal epidural steroid injections in low back pain: A one-year experience. Pain Physician 2002; 5:266-270.

11. Schofferman J, Reynolds J, Herzog R et al. Failed back surgery: Etiology and diagnostic evaluation. Spine / 2003; 3:400-403.

12. Long DM, Filtzer DL, BenDebba $M$ et al. Clinical features of the failed-back syndrome. J Neurosurg 1988; 69:61-71.

13. Slosar PJ. Indications and outcomes of reconstructive surgery in chronic pain of spinal origin. Spine 2002; 27:2555-2562.

14. Phillips FM, Cunningham B. Managing chronic pain of spinal origin after lumbar surgery: the role of decompressive surgery. Spine 2002; 27:2547-2553.

15. Van Goethem JW, Parizel PM, Jinkins JR. MRI of the postoperative lumbar spine. Neuroradiology 2002; 44:723-739.

16. North RB, Ewend MG, Lawton MT et al. Failed back surgery syndrome: 5 -year follow-up after spinal cord stimulator implantation. Neurosurgery 1991; 28:692-629.

17. Prager JP. Neuraxial medication delivery: the development and maturity of a concept for treating chronic pain of spinal origin. Spine 2002; 27:2593-2605.

18. Holst D, Mollmann M, Schymroszcyk B et al. No risk of metal toxicity in combined spinal-epidural anesthesia. Anesth Analg 1999; 88:393-397.

19. Racz GB, Sabonghy M, Gintautas J et al. Intractable pain therapy using a new epidural catheter. JAMA 1982; 248:579-581. 
